Österreichische Akademie der Wissenschaften / Austrian Academy of Sciences AAS WORKING PAPERS IN SOCIAL ANTHROPOLOGY

Volume 11

Elisabeth Katschnig-Fasch

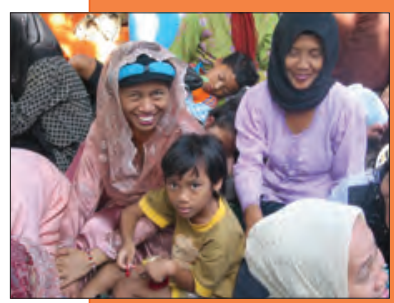

\title{
Das Janusgesicht des neuen kapitalistischen Geistes
}

Intulath

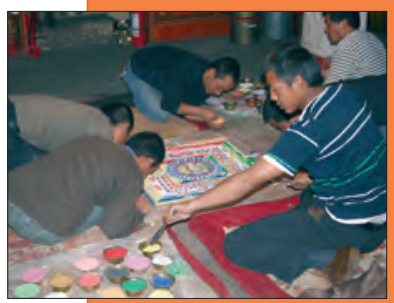

Band 11 


\section{AAS Working Papers in Social Anthropology / \\ ÖAW Arbeitspapiere zur Sozialanthropologie}

ISSN: 1998-507X

doi: 10.1553/wpsa11

ISBN: 978-3-7001-6711-2

Wien 2010

\section{Editors / Herausgeber:}

Andre Gingrich \& Guntram Hazod

(C) Institut für Sozialanthropologie

Zentrum Asienwissenschaften und Sozialanthropologie

Österreichische Akademie der Wissenschaften

Prinz-Eugen-Straße 8-10

A-1040 Wien

Fax: 01/ 51581-6450

E-Mail: sozialanthropologie@oeaw.ac.at 


\title{
DAS JANUSGESICHT DES NEUEN KAPITALISTISCHEN GEISTES
}

\author{
Elisabeth KaTSChNIG-FasCH * \\ Universität Graz
}

Mit ungeheurer Vehemenz und Radikalität wurde in den vergangenen zweieinhalb Jahrzehnten unter neoliberaler Dynamik das Gefüge unserer Gesellschaft umgebaut, wobei sich die Lebenssituation und die Alltagsbefindlichkeit vieler verschlechtert haben. Atypische Arbeitsverhältnisse, Patchwork-Arbeitskarrieren, Junk-Jobs, die dazu zwingen, dass immer mehr mehrere Jobs haben müssen, um überhaupt über die Runden zu kommen, Zeitarbeitsverträge ohne arbeitsrechtliche Sicherheit und ohne Ansprüche, brüchige Karrierewege, Studienabschlüsse ohne Garantie; das Niveau der Arbeitslosigkeit ist hoch und steigt, die Population an und unter der Armutsgrenze wächst.

An einigen Aspekten soll hier gezeigt werden, wie die marktmäßigen Mechanismen, die zur Prekarisierung der Arbeitswelt führten, von lebensweltlich bedingten Mechanismen unterfüttert, unterschiedlich wahrgenommen und verarbeitet werden. ${ }^{1}$

Der Neue Geist des Kapitalismus (nach Chiapello \& Boltanski 2003) kam hierzulande im Vergleich zu westeuropäischen Industriestaaten verhältnismäßig spät in Schwung. Bis in die 2. Hälfte der 1980er Jahre waren Arbeitnehmer/innen in der österreichischen Struktur der Kleinund Mittelbetriebe und im ausgebauten Wohlfahrtsstaat gut eingebettet. Mit dem Umbau zur postfordistischen Arbeitswelt und dem Einzug neoliberaler Marktwirtschaft begann das bis dahin gut verankerte Leitbild sozialstaatlich regulierter und sicherer Lohnarbeit zu bröckeln. Da es nun immer deutlicher darum ging, die Vorherrschaft des freien Marktes zu sichern, hatten sich die Menschen auch hierzulande an der Norm der neoliberalen Umstrukturierung auszurichten und vor ihr zu bestehen. Anthropologisch gesehen bedeutet diese Entwicklung, dass der Mensch flexibel zu sein und sich fluiden Verhältnissen anzupassen hat. Das meint sowohl das Verhältnis zu Raum, zu Zeit und Arbeit, wie auch sein Verhältnis zu sozialen Gefügen, ${ }^{2}$ was neue Einstellungen und neues Handeln erfordert. Zeit muss arbeiten, Rastlosigkeit und Aktivität bestimmen den Alltag, die Grenzen zwischen Produktion und Reproduktion werden ununterscheidbar, Privatheit und Öffentlichkeit sind nicht mehr zu trennen, Kreativität wird zur Pflicht. Der Mensch ist zum selbst

\footnotetext{
* Der folgende Artikel basiert auf einem Vortrag, den die Autorin am Institut für Sozialanthropologie (ISA) der Österreichischen Akademie der Wissenschaften im Rahmen der ISA International Guest Lecture gehalten hat (Wien, 15. 10. 2009).

${ }^{1}$ In zwei Forschungsprojekten während des Zeitraums 1999-2008 haben wir Menschen aus unterschiedlichsten Berufen, Milieus und Alterszugehörigkeiten nach ihren Erfahrungen und ihrem alltäglichen Umgang mit den Umbrüchen befragt und ihre Lebensbefindlichkeiten unter den Bedingungen der fortschreitenden Prekarisierung untersucht. In beiden Projekten - Das ganz alltägliche Elend. Begegnungen im Schatten des Neoliberalismus, hg. v. Elisabeth Katschnig-Fasch (2003), eine Anschlussforschung zu Pierre Bourdieus großer Studie La misère du monde (1993, dt.: Das Elend der Welt 1997) und im Forschungsprojekt zur Situation prekariserter Jugendlicher (Gerlinde Malli, Gilles Reckinger, Diana Reiners, Müssen nur wollen. Eine Bestandsaufnahme der Situation prekarisierter Jugendlicher, 2005-08) lag der Fokus auf der gegenwärtigen österreichischen Befindlichkeit.

${ }^{2}$ „,Der Kontaktaktmensch besitzt sich selbst, in so ferne er selbst das Produkt seiner eigenen Arbeit an sich selbst ist“" (Boltanski und Chiapello 2003: 208).
} 
verantwortbarem Leistungsvermögen gezwungen, zum Humankapital geworden, die gesamte Person zum Produktionsprozess, so der Sozialphilosoph André Gorz (2004: 20). Unter hohem Subjektivierungszwang und unter dem Druck, sich ständig neu zu organisieren, umzuschulen, weiterzubilden, sich am Laufen zu halten, wurde er umgeformt. Mit Fug und Recht ist in diesem Zusammenhang von einer Politik zu sprechen, die eine regelrechte Metamorphose des Menschen eingeleitet hat, da sie entsprechende funktionale Eigenschaften des Körpers einfordert, auch neue mentale und psychische Eigenschaften, die Fähigkeit, auf Neues rasch zu reagieren, selbstverantwortlich und flexibel zu sein, auch möglichst bindungslos zu leben. Um diesen Vorgaben zu entsprechen, ist es notwendig, sich von verinnerlichten, habituell erworbenen und auf Langfristigkeit und Beständigkeit ausgerichtete Haltungen und Wertmustern, ebenso wie von milieu- oder standesspezifisch verankerten Verhaltensmustern und Einstellungen zu lösen. Das kann zwar auch Befreiung bedeuten, wobei sich dieser Aspekt der Individualisierungsthese uns allerdings selten bestätigen konnte. Stattdessen kommt dies als Zwang daher und als Entfremdung vom Eigenen, was eine regelrechte Abwertung der verinnerlichten Wertmuster verlangt. Das wiederum bedeutet Selbstentwertung, womit sich die gegenwärtige Kultur in einer besonders destruktiven Weise ins Spiel bringt.

Während bis in die 2. Hälfte des vorigen Jahrhunderts die Kultur von der Ethik des Sparens gefärbt und einer Moral der Arbeitskompetenz verpflichtet war, einer Moral, die Max Weber als rationale Askese bezeichnet hat (1988), und die mit Solidarität auf Gegenseitigkeit und Erfahrungswissen nach dem Grundsatz „Leistung gegen Teilhabe“ vor dem Hintergrund garantierter Normalarbeitsverhältnisse verbunden war, so ist diese Basis in einer „Kultur des neuen Kapitalismus“ (Sennett 2006) zerbrochen, stattdessen ist sie durch die Enttäuschung der Erwartungen von Unsicherheit und Verwundbarkeit besetzt. Eine Kultur der Krisen, nicht nur ökonomisch oder ökologisch, sondern auch ideologisch: vom Kollektivinteresse zu Profitinteresse, von sozialer Verantwortung zu Wettbewerb. In der Gefangenschaft des Einzelnen zwischen der Aufforderung zur Selbstbestimmtheit angesichts (angeblich unendlicher) neuer Möglichkeiten und gleichzeitig noch nie da gewesenen Zwängen wirkt das Janusgesicht des neuen kapitalistisches Geistes.

Die Frage ist, mit welchen Konzepten wir diese alltäglichen Krisen beschreiben und verstehen können. Bekanntlich kursiert bereits ein ganzes Kaleidoskop an Begriffen: Abgehängte, neue Armut, Überflüssige, Überzählige, Randgruppen, Underclass, Unterschicht, Working poor... . Aber alle Benennungsversuche sind vorbelastet, auch ambivalent und allemal aus den Angeln zu heben. Armut, so meinte man noch zu Beginn unserer Untersuchungen, gäbe es in Indien, in Afrika, aber nicht in westlichen Ländern, die zu den reichsten zählen. Und von Unterschichten zu sprechen, so der Einwand, entspräche wiederum nicht der political correctness, sei beleidigend für Leute, als sei die Bezeichnung die Beleidigung und nicht der Zustand, in dem sie sich befinden. Jede Etikettierung ist jedenfalls auf ihre Art problematisch, nicht zuletzt weil sie Gestaltungsmacht für die Durchsetzung von Diskursen erlangen, also auch Teil des Ungleichheitsgeschehens werden kann. Tatsächlich treffen die Bezeichnungen nicht das eigentlich Problem unserer Gegenwart, nämlich die Unsicherheit, die sich in allen westlichen Industrieländern breit gemacht hat. Das Konzept der Prekarität eignet sich daher für die kulturwissenschaftliche Analyse besonders, nicht zuletzt deshalb, weil damit der Prozess des Umbruchs und seine Auswirkungen auf die Realität und Lebensbefindlichkeit der Menschen in den Blick genommen werden kann. Prekarisierung, wie 
Bourdieu formuliert, bedeutet eine „Destrukturierung des seiner zeitlichen Strukturen beraubten Daseins und dem daraus resultierenden Verfall jeglichen Verhältnisses zur Welt, zu Raum und Zeit“ (Bourdieu 1998: 97). ${ }^{3}$ Seine These „Prekarität ist allgegenwärtig“ mag zwar plakativ erscheinen, aber sie ist richtig. Ungewissheit beschreibt ein Gefühl, das zudem keinesfalls denen vorbehalten ist, die bereits abgehängt sind, sondern stellt eine zentrale Qualität der gegenwärtigen Lebensbefindlichkeiten dar. ${ }^{4}$

In der Weise, wie der Begriff Prekarität heute verwendet wird, taucht er sehr spät auf. ${ }^{5}$ Das mittelalterliche Prekarus bezeichnet im juristischen Sinne das, was allein durch Gebet erlangt werden kann. Das dürfte für sehr viele Menschen, die in diesem Zustand sind, zutreffend sein und die relevante Tonlage dessen, was prekär bedeutet, stimmend einfangen. Seine große Karriere begann der Begriff Prekarität in Frankreich der 1980er Jahre. Man sprach von Neuer Armut, das Mindesteinkommen wurde erstmals eingeführt, die Erfahrungen und der gesellschaftlichen Umbruch wurden virulent. ${ }^{6}$ In Österreich wurde der Begriff Pekarität erst in den späten 1990ern geläufig, zunächst als Äquivalent zu atypischen Arbeitsverhältnissen, später bezeichnet der Begriff zudem eine Risikokonstellation und spezifische, belastende Arbeitsbedingungen. In Anlehnung an den Begriff Proletariat wurde Prekariat erfunden, ein Terminus, den zunächst Kreativarbeiter, Künstler und in der IT- Branche Tätigen für sich beanspruchten, also diejenigen, die aus dem Herrschaftsregiment der fordistischen Arbeitswelt ausbrechen wollten und bewusst auf Individualisierung der Arbeitswelt setzten und gelegentlich auch profitieren konnten. ${ }^{?}$

Uns ging es um jenen Prozess der Prekarisierung, in den Menschen durch die postfordistische Rationalisierungspolitik geraten und der das Ganze ihres Lebens erfasst. Dieser Prozess spielt sich gleichzeitig auf zwei Ebenen ab: Auf objektiver/struktureller Ebene als Schwächung des Status durch die Prekarisierung der Arbeitswelt und der materiellen Lebensverhältnisse - etwa durch die Abwertung von schulischem Kapital und die Deregulierung rechtlicher Gewissheiten -, sowie auf der kulturellen Ebene, jener der Effekte in der Alltagswelt - Entfremdung, Verletzung der Würde, Enttäuschung der Erwartungen, Verlust von Planbarkeit. Wir suchten Menschen über längere Zeiträume in ihren prekarisierten Existenzbedingungen auf und sprachen mit ihnen. Was zum Ausdruck gebracht wurde, galt es dann in seiner inneren und äußeren Beziehungspraxis zum Verstehen zu bringen. Nicht bloß „Hineinversetzen“ ist damit gemeint, sondern ein genetisches Verstehen, um die sozialen Strukturen und kulturellen Prinzipien, die hinter den Erfahrungen der Menschen stehen, fassen zu können. Dies entspricht einem Kulturbegriff, der, wie er etwa auch

\footnotetext{
${ }^{3}$ Prekarität hat für den, der sie erleidet, tief greifende Auswirkungen. Indem sie die Zukunft im Ungewissen lässt, verwehrt sie den Betroffenen zugleich jede rationale Planung und damit ,jenes Mindestmaß an Hoffnung und Glauben an die Zukunft, was für eine vor allem kollektive Auflehnung gegen eine unerträgliche Gegenwart notwendig ist" (Bourdieu 1993: 97).

${ }^{4}$ Ich danke Franz Schultheis für anregende Gedanken aus seinem Vortrag "Prekarität und Flexibilität: Die zwei Gesichter der sozialen Frage der Gegenwart", den er am Institut für Volkskunde und Kulturanthropologie der Universität Graz im April 2009 gehalten hat.

${ }^{5}$ Eher beiläufig findet sich der Begriff bei Karl Marx in Kritik der politischen Ökonomie als Zustand dessen, was prekär ist (vgl. Seifert 2009).

${ }^{6}$ Damals hat P. Bourdieu das Konzept Prekarité in seinem berühmten Buch La misère du monde für den Übergang der industriellen Gesellschaft Frankreichs in die neoliberale exemplifiziert (Bourdieu 1997).

${ }^{7}$ Der amerikanische Wirtschaftswissenschaftler Richard Florida spricht ganz euphorisch von einer neuen Kreativ-Klasse (Barth 2009).
} 
von Raymond Williams als ,the whole way of life“ gefasst, wirtschaftliche, soziale, politische und historische Voraussetzungen inkludiert (vgl. Lindner 2006).

Zur Veranschaulichung der Umgangsstrategien greife ich auf einige Beispiele zurück. Es sind dies Beispiele aus Milieus der „,unteren Mitte“, jenen Bereichen, in denen soziologische Analysen die eigentlichen Verlierer orten. ${ }^{8}$ Diese Exempel stehen paradigmatisch für unterschiedliche Praxisformen des Umgangs und zeigen, wie stark die Erfahrung, die Wahrnehmung und der Umgang mit prekären existentiellen Bedingungen vom jeweiligen soziokulturellen Kontext, von Relationen, Wahrnehmungsweisen und von inkorporierten Werten bestimmt wird. ${ }^{9}$

Wenn beispielsweise ältere Gesprächspartner, deren Biographie lange in traditionell vorgegebenen Arbeiterlaufbahnen gebettet war, erzählten, dann so, als erlebten sie ihre Degradierung nicht als eine von außen verursachte Ungerechtigkeit. Sie erzählten von ihrer Abkoppelung, vom Verlust von Anerkennung und von Planungsmöglichkeit der eigenen Zukunft, vom Verschwinden kollektiver Identität, von Wertewelten, die sie nicht mehr verstehen, von Identitätskonflikten und ihrer psychischen und physischen Verwundbarkeit. Sie alle schrieben sich die Situation selbst zu, so, als ob sie selbst das gesellschaftliche Leitbild beschädigt hätten. Das, was sie eigentlich verletzte oder beeinträchtigte blieb dabei im Diffusen, was aber bedeutet, dass der vorenthaltenen Anerkennung und der erlebten Degradierung auch keine Chance zum Widerstand, zum Schutz der eigenen Integrität bleibt (vgl. auch Honneth 2000: 180). Die Ursachen des Unbehagens und der Verletzung wanderten ins Unbewusste ein, sie wurden verdrängt.

Ich denke an einen heute 67-jährigen Facharbeiter eines Metall verarbeitenden Betriebes, der in den 1980ern gegen die erste große Kündigungswelle noch politisch kämpfte und in der sukzessiven Erfahrung der Ohnmacht die Fahrzeuge der Managerriege aus Rache „umzudrehen“ gedachte - damals noch aus der Position eines Angehörigen der Arbeiteraristokratie, der Werkmeister. Dann kamen CNC-Maschinen und eine neue Generation junger Techniker. Handwerkliches Wissen und Erfahrung waren plötzlich unnütz, der gewerkschaftliche Hintergrund zerbröckelte. Hunderte Arbeitsplätze gingen verloren, was für ihn und für die älteren Kollegen, die nicht damit gerechnet hatten, eine dramatische Ernüchterung bedeutete, von der sich viele nicht mehr erholen konnten. Als ich ihn Jahre später noch einmal aufsuchte, fand ich ihn nicht nur ohne Arbeit und geschieden, auch ohne Worte der Klage, depressiv und ohne jegliches Interesse an der Politik.

Es ist das Privileg der Arbeitsgesellschaft gewesen, fest in Erwerbsarbeit eingebunden gewesen zu sein. Die Menschen verfügten daher auch über ein kohärentes System von Erwartungen und Forderungen, auch über eine kohärente Vorstellung des sozialen Raums und über seine Regeln darin. Als die gewohnten Strukturen nicht mehr gegeben waren, konnten diejenigen, die noch orientiert und ausgerichtet auf die Normalität einer fordistischen, wohlfahrtsstaatlichen Arbeitskultur waren, auch kein Handeln nach der neuen Logik generieren. Viele blieben in

\footnotetext{
${ }^{8}$ Michael Vester unterscheidet den alten Mittelstand (Kaufleute, Handwerker, Landwirte), der bis 2000 von 25\% auf 5\% schrumpfte, und den neuen Mittelstand der Angestellten, dem sich die Industriearbeiterschaft annäherte, in der die alten Merkmale des Proletariats schwanden und die die sozialen und kulturellen Standards der sozialen Mitte erreichten (Vester 2009).

${ }^{9}$ Schon Emile Durkheim hat darauf verwiesen, dass Milieus sich gleichzeitig „objektiv“ durch Berufsfelder und „subjektiv" durch ihren Habitus definieren (Durkheim 1988); auch Max Weber argumentiert, dass sich die sozialen Alltagszusammenhänge durch ihre gesamte Praxis von einander abgrenzen, ihnen also auch praktische Differenzen zugrunde liegen, ästhetische, ökonomische wie eben auch Unterschiede in der Lebensführung des Alltags (Weber 1972: 236).
} 
Resignation hängen, was jedoch keineswegs auf eine subjektive Reaktion zu reduzieren ist, sondern zum Habitus der älteren Generation von Facharbeitern geworden ist.

Ein anderes Reaktionsmuster auf die Erfahrung der Ersetzbarkeit zeigt bereits die nächste Generation: Stellvertretend das Beispiel eines heute 45-jährigen Werkzeugmachers. Auch er dachte anfangs sein Leben lang im gleichen Betrieb zu arbeiten wie der Vater und der Großvater vor ihm. Das hat sich für ihn schnell als Irrtum erwiesen. Kaum eingestellt, wurde er, von Maschinen ersetzt, gekündigt. Heute ist sein Handeln nicht einer Solidarität auf Gegenseitigkeit verpflichtet, im Gegenteil. Um den geringer gewordenen Verdienst auszugleichen, setzt er auf Abfertigung. Immer wieder. So wechselt er von einem Job in den anderen. Er kalkuliert und schwimmt auf diese Weise mit der Welle der Flexibilisierung mit und versuchte sich den neuen Bedingungen soweit anzupassen, dass sie gewissermaßen zu seinem Charakter geworden sind, mit dem er zwischen Kurzeit und Teilzeitjobs jongliert. Er hat gesundheitliche Probleme und sieht sich nicht in der Lage, gemeinsam mit seiner Lebensgefährtin für eine einigermaßen gesicherte Zukunft auch nur eines einzigen Kindes zu sorgen. Ist sein Verhalten Widerstand oder Anpassung? Auch hier zeigt sich die Doppelgesichtigkeit des neuen Geistes. Sein Umgang mit den Strukturen steht nicht im Zeichen einer widerständigen Neubewertung, sondern im Kontext des hegemonialen Regimes. So bleibt sein Handeln als „Waffe“ zu schwach, um das System zu schlagen. Er verliert, trotz seiner Bemühungen, der Abwärtsspirale zu entkommen. Als einer jener „Verlierer der ökonomischen und technologischen Modernisierung“ (Vester), die ihre Situation mit Ressentiments beantworten, sucht auch er die Ursache seiner Misere bei den billigen Arbeitskräfte aus Osteuropa, den Ausländern und bei den Politikern. Von seiner politischen Heimat hat er sich gelöst, jetzt sympathisiert er mit den Rechten. ${ }^{10}$

Unsichere Arbeitsverhältnisse machen Loyalität und Solidarität unsinnig. Sie führen jenen, die noch in gesicherten Bedingungen arbeiten, die Entwertung des Wissens und deren soziale Deklassierung vor Augen und machen ihnen bewusst, dass auch sie keine Zukunft haben. Individuelle Arbeitsverträge mit individueller Entlohnung und Bewertung, Sonderleistungen und Zielvorgaben bedeuten Positionsunsicherheit und führen zu Misstrauen, ${ }^{11}$ wobei das Band der Arbeitersolidarität oft mitten in der eigenen Familie zerreißt. Hier tut sich nicht nur eine identitäre Spaltung im Einzelnen auf, durch die kulturelle Zeitinkohärenz zudem auch eine generative. Die Älteren sehen zerstört, wofür sie lange gestritten und gekämpft haben. Sie begreifen die Jüngeren nicht, die in ihren Augen verlernt haben, als „richtige Arbeiter“ zu denken. Durch Flexibilisierung und Deregulierung schwindet die Epoche der verbindlich organisierten Arbeit und damit die gemeinsame kulturelle Lage. „Ein im historischen Lernprozess gefestigter und habituell inkorporierter, sozialer und kultureller Kapitalbesitz ist nicht mehr reproduzierbar“ (Franz Schultheis). ${ }^{12}$ Das, was bereits Max Weber als den wesentlichsten Faktor der Kultur festmachte, das Bewusstsein einer solidarischen Zugehörigkeit, ist unter den Bedingungen der konkurrenzierenden Marktwirtschaft jedenfalls dahin.

${ }^{10}$ Ein Phänomen, das M. Vester ebenso für die Bundesrepublik Deutschland konstatiert (Vester 2009a).

${ }^{11}$ Dies wird durch den Diskurs über die noch vorhandenen „Privilegien“ der jeweils anderen verstärkt.

${ }^{12}$ Ein weiteres Faktum, das ebenso in die sich auftuende Generationenspaltung nahtlos eingeht, ist der Ruf nach immer längerer Lebens-Arbeitszeit auf der einen und die Realität der Unternehmensökonomie auf der anderen Seite. Alle wissen, dass die immer weniger werdenden Jüngeren die Sicherungssysteme nicht mehr finanzieren können. In dieser Zwangslage empfinden die Jüngeren es als Zumutung für die Alten zahlen zu müssen. 
Ursache einer Befindlichkeit, die vielen zur unerträglichen Last geworden ist, kann also keineswegs auf erhöhte Arbeitsbelastung reduziert werden, vielmehr ist es die machtvolle Verunsicherung, die wie in einer unaufhaltsamen Kettenreaktion Arbeitnehmer wie Arbeitgeber, Frauen wie Männer, Junge wie Ältere trifft und nicht allein auf traditionelle Arbeitermilieus beschränkt ist. Wenn auch in unterschiedlichem Ausmaß - so ist davon die gesamte Mittelstandsgesellschaft betroffen. Ihr gesichert angenommenes Stellenrevier ist geschwunden, der Zusammenhang zwischen Herkunft und Bildung, zwischen Bildungsabschluss und Anstellung und zwischen Anstellung und Entlohnung ist zerbrochen, die Weitergabe des sozialen Erbes nicht mehr garantiert. Zwischen einer Zukunft, die nicht zu erreichen und einer Vergangenheit, die nicht anschlussfähig ist, hat sich das Leben auch in der „respektablen Mitte“ (Vester 2009) zu einer Abfolge von Zwängen, Druck und Überforderung verwandelt, nicht aus der Verelendungslogik, sondern aus ihrer Gerechtigkeitslogik. Die gewohnten Werte der Achtung und Selbstachtung sind in Frage gestellt, die hohe Leistungsbereitschaft ist keine Gewährleistung mehr. Zwischen dem Anspruch auf Sicherheit und Wohlstand und der Angst, den Maßstäben nicht mehr genügen zu können, zwischen den in Österreich besonders tief verinnerlichten Erwartungen einer Statusstabilität einerseits und den prolongierten Anforderungen von Flexibilität, Risikobereitschaft und Verfügbarkeit andererseits stecken die Menschen auch in bisher als sicher angenommenen Milieus fest.

Das Gefühl des Gefangenseins wirkt umso schwerwiegender, je elementarer die Veränderungen den Habitus treffen. An sich schafft der sozial und kulturell erworbene Habitus eine stimmige Verankerung des Einzelnen und damit Sicherheit der Identität, verfestigt in der Mentalität und Haltung (Bourdieu 1983). Wenn aber Erwartungen, Einstellungen und Lebensziele von der Realität neuer struktureller Gegebenheiten und neuer Wertsysteme überrollt werden, verschwimmen die Orientierungen. ${ }^{13}$ Auch wenn durch den Wandel der Sichtweisen der habituelle „Modus der Evidenz“ in Frage gestellt werden und sich verändern kann, müssen Veränderung des Bewusstseins von innen ausgehen. Wo erwartete soziale Absicherung zurückgefahren wird, die Sicherheiten zurückgenommen werden, der Status gefährdet ist und befristete Beschäftigung oder sogar Arbeitslosigkeit droht, führt dies zu einer Desorganisation des Verhaltens, die ihrerseits eine Orientierung auf neue Bedingungen und neue Zeithorizonte hin behindert. Das Leben bleibt unter dem Eindruck des Provisorischen und damit der Inkohärenz zwischen neuen Erfordernissen und erlernten Verhaltensweisen ausgeliefert (Bourdieu 2000: 107). Daraus resultieren Selbstentwertung, auch Gefühle von Überforderung, Unsicherheit und Ängste, was es schwer möglich macht, sich den Wertkategorien einer Kultur der Ungewissheit bedingungslos zu unterwerfen (vgl. auch Baumann 1999: 127). Letztlich ist es die Hoffnung, es schon schaffen zu können, die zumindest Jüngere noch vor Verzweiflung bewahren kann, die aber gleichzeitig auch dafür sorgt, dass in ihrer kulturell gut verankerten meritokratischen Logik auch ihr Schuldgefühl nicht versiegt.

Begegnungen in der überwiegend von Frauen dominierten Berufsgruppe der Reinigungskräfte zeigten uns jedoch, dass ein unsicherer und belasteter, prekärer Arbeitsplatz nicht auch gleichzeitig ein prekäres Leben bedeuten muss. Ausgerechnet niedrig bezahlte Putzfrauen führten uns vor Augen, wie kurz gegriffen es prinzipiell ist, die objektiven sozialwissenschaftlichen Prekariatstypologien

${ }^{13}$ Bourdieu spricht von eine regelrechte „Gespaltenheit“" als Folge erlebter Unvereinbarkeiten, ob in der Arbeitswelt oder in der Familie. Es charakterisiert das aus dem Gleichgewicht geratene Verhältnis zu Zeit, Gestern und Heute, unvereinbarer kultureller Wertsysteme. Damit manifestiert sich die Ungleichzeitigkeit in verschiedenen Bewusstseinslagen, womit die Person im Inneren gespalten ist und eine permanente Andersartigkeit ihrer Subjektivität erfährt (Bourdieu 1997: 237). 
zum alleinigen Maßstab der Erfahrung von prekärem Leben zu nehmen, wie wichtig stattdessen neben der Relation der habituellen Erfahrung, das Motiv des Gestaltungsspielraums und das Gefühl der sozialen Eingebundenheit und Gegenseitigkeit als Ressource für das Gelingen eines eigenen Lebens ist. ${ }^{14}$

Zur Veranschaulichung: Unsere 41jährige Protagonistin arbeitet als Reinigungskraft großer Gebäudereinigungsfirmen. Wie die meisten in ihrer Putzkolonne ist sie im ländlichen Umfeld aufgewachsen. Nun lebt sie mit ihren zwei Kindern in der Stadt. Angesprochen auf die Arbeitsbelastung setzte sie zu unserer Überraschung gleich ihre Zufriedenheit vor weg. Ihr gäbe die Arbeit immerhin die Möglichkeit, nach einer belasteten Ehe sich endlich ein eigenes Leben zu schaffen. In von Zeitknappheit diktierter Selbstdisziplinierung und unter dem Druck von ständiger Kontrolle und entsprechenden Sanktionen motiviert sie sich und ihre Kolleginnen mit der Überzeugung, dass es darum gehe, darum zu kämpfen, dass ,alles hinhaut, sonst hat ja keine von uns einen Job“. Angepasst an die objektiven Möglichkeiten, die ihrem Habitus und ihrem weiblich intonierten Realitätssinn entsprechen, einer Realität des Notwendigen und der Zweckmäßigkeit, sprach sie dennoch von ihrer Selbstbestimmtheit, von Solidarität der Kolleginnen und auch vom Stolz, für eine große Versicherungsanstalt zu putzen, von ihrem Stück Zeitsouveränität, von ihrer Verantwortung und vom Gefühl gebraucht zu werden: „Wie würd' denn die Welt ausschauen, wenn es Putzfrauen nicht gäbe?" Wie sie haben ihre Kolleginnen den Zwang verinnerlicht und begründen ihn als Pflichterfüllung, als Pflicht, die ihrem Leben Sinn gibt und die ihre Lebenssituation nicht unerträglich macht. Das Motiv liegt im kollektiven Gedanken und in der Gewissheit, dass alles so kommt, wie es am besten ist. Dieser Schicksalsglaube ist nicht Fatalismus, der zur Passivität führt, vielmehr kompensiert er die objektive Unsicherheit dieses prekären Berufsfeldes und ist als Glaube an die „Vorsehung“ nach Max Weber sogar psychischer Antrieb (Weber 1988). Offensichtlich glauben auch heute noch immer Menschen daran, ,,von oben“ dorthin gestellt zu sein, wo sie stehen und wo sie ihre Pflicht zu erfüllen haben. Durch Generationen eingeübte Strategien auf existentielle Unsicherheit haben sie vorbereitet und eingeübt, flexibel zu sein und verschiedene Erwerbstätigkeiten zu kombinieren oder Demütigungen zu verarbeiten, bereit auch, gering bezahlte Arbeiten anzunehmen und Belastungen auszuhalten. ${ }^{15}$

So paradox es angesichts der prekären Arbeitslage dieser Frauen erscheinen mag, gerade die traditionelle, geschlechts- und milieutypische Prägung einer „Sinn-Ökonomie“, die von nachwirkenden Gewohnheiten und Einstellungen noch gehalten wird, schützt in der Alltagsrealität gegenwärtiger Profitmaximierungsideologie eben das, was der Kapitalismus nicht bieten kann, den Sinn des eigenen Lebens. So lange Arbeit als schöpferisches Tun verstanden werden kann, bleibt auch der Lebenssinn erhalten - eine entscheidende Ressource, um die Effekte der Individualisierung nicht negativ erfahren zu müssen. Eine solche Sinn-Ökonomie steht nicht im Einklang mit der Ideologie des Zeitgeistes. Aber - und auch das ist Wirkung der ungeschminkten Doppelgesichtigkeit

${ }^{14}$ Die transformierbaren Kapitalien - ökonomisches Vermögen, akkumuliertes soziales und kulturelles Kapital - folgen in den unterschiedlichen Feldern auch je eigenen Logiken (Bourdieu 1983; siehe dazu auch Seifert 2009).

${ }^{15}$ Abgesehen davon, dass hier einmal mehr der Vorwurf widerlegt ist, dass das soziale Netz eher missbraucht, als dass gering bezahlte Arbeit angenommen werde, zeigt sich daran, wie stark die von Weber beobachteten ständigen Praxisformen heute noch immer Klassenunterschiede mitbestimmen. Angehörige unterprivilegierter Milieus sind sich der Gefahr bewusst, stigmatisiert zu werden. Sie tun auch viel, um einer sozialen Ausgrenzung, vor allem auch ihrer Kinder, vorzubeugen. Es geht nicht allein um das Überleben, sondern um das Mithalten (Vester 2009). 
des neuen kapitalistischen Geistes - die Logik der Gewinnmaximierung funktionalisiert gerade diejenigen besonders effektiv, die noch im kollektiven Habitus verankert als billigste Arbeitskräfte ihr Überleben noch irgendwie sichern können und dabei unbewusst zu ihrer eigenen Unterdrückung beitragen. Ausbeutung und Selbstermächtigung liegen nahe beisammen, ununterscheidbar bisweilen.

Sinnvolles Handeln und sinnvoll erlebtes eigenes Leben vollzieht sich nur im „Horizont des Anerkannt-Seins“ (Paul Ricoeur 2006), eine Tatsache, die für Jugendliche aus prekaristerten Familien ganz an Brisanz gewonnen hat, da sie vom wirtschaftlichen Umbau in besonderer Weise getroffen sind.$^{16}$ Die Sozialisation dieser jungen Menschen steht anders als die der Elterngeneration bereits auf dem schwankenden Untergrund der Prekarität. Sie sind mit Benachteiligung und familiären sozialen und psychischen Belastungen, Arbeitslosigkeit und wechselnden, unsicheren Beschäftigungen der Eltern aufgewachsen. Sie haben die neuen Werte des gegenwärtigen kapitalistischen Geistes bereits inkorporiert: Sie sind flexibel, sie sind mobil, fühlen sich nicht mehr an Loyalitäten gebunden und sie sind auf ihr eigenes Überleben fokussiert. Sie sind also bereits angepasst und können dennoch oder gerade deswegen die prekären Strukturen nicht überwinden. Erik Erikson sieht vor allem im Zerfallen der primären Institution Familie den zentralen Grund dafür, dass es Jugendlichen nicht gelingt, Ressourcen und Praktiken zu mobilisieren, die für das soziale Überleben notwendig sind (Erikson 1993). Das Fehlen der elementaren Sicherheit erschüttert das Selbstvertrauen und zerstört ihre Identitätsfindung. Eine sekundäre Identität zu konstruieren verunmöglicht sich ihnen wiederum durch den zunehmenden Zerfall gesellschaftlicher Verantwortung. Die Antwort prekarisierter Jugendlicher darauf: sie drehen ihre Position sozusagen um und gestalten sich selbst zu ,aktiven Verweigerern“, um dann der Arbeitsgesellschaft bewusst den Rücken zu kehren und in sozialstaatlichen Abhängigkeiten hängen zu bleiben. Sie haben gelernt, sich durchzuwursteln und ihre Scham hinter der Maske einer lauten Forderung nach Sozialhilfe zu verbergen, um irgendwie über Wasser zu bleiben. Der Investition in eine Zukunft können sie nichts abgewinnen. Sie misstrauen den Erwachsenen, die wiederum ihre Probleme haben, sie zu motivieren. Und wenn diese Jugendlichen dann vorzeitig die Schule abbrechen und das Feld räumen, nach dem sie alle Angebote unterlaufen haben, empfinden Lehrer oft nur noch Erleichterung. Die vielen gescheiterten Versuche, sich in die Arbeitsgesellschaft zu integrieren oder die vielen, in ihren Augen sinnlosen Arbeitslosenmaßnahmen sind ihnen bloß Bestätigung dafür, wie wenig man sie ernst nimmt und wie wenig die Gesellschaft auf sie Wert legt. Anerkennung wird ihnen in ihren Augen bewusst verweigert.

Den Jugendlichen fehlt, und das ist gegenüber der Elterngeneration neu, der Wille zum Aufstieg. Er ist ihnen angesichts der familiären Prekaritätserfahrung abhanden gekommen. Das Bildungssystem halten sie für einen Betrug, Bildungsabschlüsse für wertlos. Sie vertrauen lieber dem Risiko. Das Gespür für gesellschaftliche Regeln und ihren Sinn haben sie verloren und damit eben auch die Einsicht in deren Notwendigkeit. Sie antworten mit Verpflichtungslosigkeit und Vertrauensverlust in die Welt der Erwachsenen und engagieren sich dort, wo sie selbst Herr ihrer Lage zu sein glauben: in alternativer Geldbeschaffung, illegaler Ökonomie, in Glücksspielen. Entwurzelt und aus der traditionellen Einbindung freigesetzt haben sie auch jeglichen klassen-

\footnotetext{
${ }^{16}$ Dieser Sachverhalt wird in der nunmehr abgeschlossenen, von der Österreichischen Akademie der Wissenschaften geförderten und in Veröffentlichung befindlichen Forschung des Doc-Teams Gerlinde Malli, Gilles Reckinger und Diana Reiners zur Lebensbefindlichkeit prekarisierter Jugendlicher Müssen nur wollen (2009) eindrucksvoll aufzeigt.
} 
oder standesspezifischen Boden verloren. In der ,grauen Ökonomie“ finden sie dann immerhin Reste von Identität. So wird Illegalität und Kriminalität von jenen Bedingungen genährt, die sie gleichzeitig sanktionieren.

Das, was ihnen offen steht, um zu Unabhängigkeit zu kommen, spiegelt die makrogesellschaftliche Entwicklung: Mobilität, Auflösung arbeitsrechtlicher Sicherung, Selbstvermarktung, Einsatz von Risiko, Spekulation. Also exakt jene Strategien, die die Marktlogik vorgibt, schreiben sich in die Handlungs- und Denklogik ein und erhöhen damit ihre vorhandene soziale Gefährdung. Statt "learning to labour" (Paul Willis 1977) - "learnung for precarity", so Gilles Reckinger. ${ }^{17}$ Das ist die Sozialisation in einer Kultur der Ungewissheit. Dass dann die Erfahrungen des Überflüssigseins, der Wertlosigkeit des eigenen Lebens bei Jugendlichen mit geringen emotionalen, ökonomischen familiären Ressourcen zu Aggression, Selbstverletzungen, Drogen und Alkoholmissbrauch und zu einer bedenklich ansteigenden Zahl von Suizidversuchen, zu Gewalt gegen andere unterprivilegierte Gruppen führt, verdankt sich eben den strukturellen gesellschaftlichen Bedingungen. Wenn Jugendliche die Grenzen der Norm überschreiten und subversiv die Freiräume ihres pubertären Moratorium nutzen und dabei ,,auf die schiefe Bahn“ geraten, dann bietet die Gesellschaft Camps in abgelegenen Naturidyllen, in Asylen der Disziplinierung. Gerlinde Malli spricht von abgesonderten mikrokosmischen Räumen, in denen sie isoliert von der Außenwelt den gesellschaftlichen Ritualen, Normen, Erwartungen und den Bedürfnissen der Erwachsenengesellschaft nach Selbstoptimierung angepasst werden. Aus dem Sozialstaat wird ein Strafstaat. ${ }^{18}$

Ungeschminkt zeigt sich das Doppelgesicht des neuen Kapitalismus somit nicht nur in der Tatsache, dass die Gesellschaft durch das Wachstum der Produktivität und des Sozialprodukts immer reicher wird, die Lagen der Mitte immer unsicherer und die Ausgrenzungen nach unten gegenüber den Überflüssigen immer deutlicher werden, sondern auch im gesellschaftlichen Umgang damit. Wir leben in einer offenen, diversifizierten Gesellschaft, gleichzeitig sind die Praktiken des Kontrollierens und Wegschließens akzeptierte Formen einer zunehmend repressiven Politik, die bestimmte Menschengruppen, ob Jugendliche, Arbeitslose oder Zugewanderte als Gefahrenquellen identifiziert. Zum einen ist dies Substrat einer Ideologie, deren innerstes Motiv Überlegenheit ist und damit auf Ausgrenzung alles dessen zielt, was nicht der funktionalen Norm des Ökonomismus entspricht, zum anderen ist es die Angst der Menschen selbst, dem Maß nicht entsprechen zu können, die das eigene und das Bedürfnis nach Sicherheit und Ordnung in einer Realität der prinzipiellen Unsicherheit mit der Abwehr des Bedrohlichen nach Außen zu sichern glaubt. ${ }^{19}$ „Die wird vor allem dann aufgesucht, wenn es zur Entfremdung des Selbst gekommen ist“ (Edward Said). Somit reduziert sich Identität auf Anpassung, was weit reichende und gefährliche Konsequenzen hat. Die Philosophin Elisabeth List spricht von einem Hyperrassismus, von einer tiefen Negation dessen, was den Maßstab der Normalität und Nützlichkeit nicht erfüllt, und von dem all jene, die nicht ,optimierbar“ oder anzupassen sind, betroffen sind. ${ }^{20}$

\footnotetext{
17 Wie Anm. 16.

18 Wie Anm. 16.

${ }^{19}$ Sigmund Freud formulierte bekanntlich diese grundlegende Konstellation immanenter Persönlichkeitsmerkmale als Bedingung gesicherter Identität.

${ }^{20}$ Elisabeth List verweist in diesem Zusammenhang vor allem auf die Ausgrenzung und Ausmerzung von Behinderung durch technische Perfektionisierung des Körpers (List 2009).
} 
Diese Logik des neuen Geistes hat sich unbemerkt über symbolische Wege, über Wort und Bild, in das Denken und Handeln eingeschlichen. So werden Opfer zugleich zu Tätern. Boltanski und Chiapello (1999) sehen im Wesen des Geistes des neuen Kapitalismus ein normatives System, dem es gelingt, Menschen immer wieder zu gewinnen und davon zu überzeugen, sich zu beteiligen. In diesem Dilemma ortet der Philosoph Alain Tourain das Hauptproblem der Modernität. Alle kulturellen Wertpositionen sind der Diskursmächtigkeit dieses neuen Geistes angepasst, dringen in die Gedankenwelt ein und unterminieren von unten die moralische Substanz des Zusammenlebens. Sie kündigen tief verankerte kulturelle Werte, wie Treue und Solidarität auf, verkehren die Wirklichkeit und ziehen unreflektiert Handlungen als Selbstverständlichkeit nach sich, die als unsichtbare Gewalt über Wege der Kommunikation ausgeübt und weiter getragen werden (Bourdieu 1997). Kern der Verfügungsmacht über Denken und Handeln ist die Ambivalenz der Begriffe. Empowerment, Eigenverantwortlichkeit, Employability, Selbstverwirklichung, Individualität und Kreativität - diese Leitbegriffe geben zwar vor, die Struktur der Disziplinierung und der Hierarchie der fordistischen Moderne zu ersetzen. In den Narrativen gesteigerter Mobilität, Flexibilität und Freiheit geht es aber nicht darum, das Leben zu demokratisieren, die Arbeit bloß zu verbessern, sondern komplett zu verändern. Dahinter verbirgt sich eine machtvolle Gouvermentalitätsstrategie als spezifisches Phänomen einer Gesellschaft, die ihre erkämpften rechtlichen Errungenschaften und kulturell gebetteten Institutionen vergessen will, beziehungsweise muss. Das, was der moderne Wohlfahrtsstaat geschafft hat, wird im Prozess der Prekarisierung zurückgenommen und re-modifiziert, womit sich der Warencharakter des Menschen als Neufassung „Humankapital“ durch die Marktvergesellschaftung mit großer Radikalität durchsetzt.

Wir haben es also mit komplexen, sozialen und kulturellen Phänomen zu tun, deren Auswirkungen in ihren unterschiedlichen, sozialen und kulturellen Dimensionen - ob in Arbeitshaltung, im Umgang mit der Zeit, dem Geld, dem Besitz, in der Selbstwahrnehmung - nicht bloß nebensächliche Anpassungen nachziehen und im Grunde vernachlässigbar sind. Im Gegenteil, sie wirken gravierend auf das Leben und müssen in ihren komplexen Zusammenhängen gerade von Kulturwissenschaften sehr ernst genommen werden.

Elisabeth Katschnig-Fasch ist Professorin am Institut für Volkskunde und Kulturanthropologie der Universität Graz. Ihre Forschungsschwerpunkte umfassen kultureller Wandel, Gender und Urbananthropologie. 


\section{Literatur}

Barth M. 2009. „Wie nennen es Kreativität“: Inszenierungen von ,alter“ und „,neuer“ Arbeit in Werbebildern der Informations- und Kommunikationstechnologie, in: Götz, Irene und Barbara Lemberger (Hg.), Prekär arbeiten, prekär leben. Kulturwissenschaftliche Perspektiven auf ein gesellschaftliches Phänomen. Frankfurt am Main: Campus, 183-204.

Baumann, Zygmunt. 1999. Unbehagen in der Postmoderne, Hamburg: Hamburger Edition.

Bolanski, Luc and Ève Chiapello. 1999. Der neue Geist des Kapitalismus (= édition discours 38). Konstanz: UVK.

Bourdieu, Pierre (1983), Die feinen Unterschiede. Kritik der gesellschaftlichen Urteilskraft. Frankfurt am Main: Suhrkamp.

Bourdieu, Pierre. 1992. Die verborgenen Mechanismen der Macht. Frankfurt am Main: VSA.

Bourdieu, Pierre. 1998. Gegenfeuer. Wortmeldungen im Dienste des Widerstandes gegen die neoliberale Invasion (= edition discourse 23). Konstanz: UVK.

Bourdieu, Pierre. 1993. La misère du monde. Paris: Edition du seul (dt.: Das Elend der Welt, Konstanz: UVK 1998).

Bourdieu Pierre. 2000. Neoliberalismus und neue Formen der Herrschaft, in: Die Augen der Herrschenden (= Isotopia 24), Graz, 12-23.

Durkheim, Emile. 1988 (1893). Über soziale Arbeitsteilung. Frankfurt am Main: Suhrkamp.

Gorz, André. 2004. Wissen, Wert und Kapital. Zur Kritik der Wissensökonomie. Zürich: Rotbuch.

Honneth, Axel. 2000. Das Andere der Gerechtigkeit. Aufsätze zur praktischen Philosophie. Frankfurt am Main: Suhrkamp.

Katschnig-Fasch, Elisabeth (Hg.). 2003. Das ganz alltägliche Elend. Begegnungen im Schatten des Neoliberalismus. Wien: Löcker.

Lindner, Rolf. 2006. Das Leben ist transdisziplinär, in: Hengartner, Thomas und Johannes Moser (Hg.), Grenzen und Differenzen. Zur Macht sozialer und kultureller Grenzziehungen. Leipzig: Leipziger Universitätsverlag, 79-88.

List, Elisabeth. 2009. Ethik des Lebendigen. Weilerwist: Velbrück Wissenschaft.

Ricoeur, Paul. 2006. Wege der Anerkennung. Frankfurt am Main: Suhrkamp.

Seifert M. 2009. Prekarisierung der Arbeits- und Lebenswelt, in: Götz, Irene und Barbara Lemberger (Hg.), Prekär arbeiten, prekär leben. Frankfurt am Main: Campus, 31-54.

Sennett, Richard. 2005. Die Kultur des neuen Kapitalismus. Berlin: Berlin Verlag.

Vester, Michael. 2009. Klassengesellschaft in der Krise. Von der integrierten Mitte zu neuen sozialen und politischen Spaltungen, in: Götz, Irene und Barbara Lemberger (Hg.), Prekär arbeiten, prekär leben. Kulturwissenschaftliche Perspektiven auf ein gesellschaftliches Phänomen. Frankfurt am Main: Campus, 55-106.

Vester, Michael. 2009a. Logiken der Milieus-Logiken der Politik. Schieflage der sozialen und der politischen Macht, Kuckuck. Notizen zur Alltagskultur „Gerechtigkeit“, 1/09, $12-17$.

Weber, Max. 1988 (1920). Die protestantische Ethik und der „Geist des Kapitalismuse, in: Ders., Gesammelte Aufsätze zur Religionssoziologie I. Tübingen: Mohr Verlag, 1-205.

Weber, Max. 1972 (1921). Wirtschaft und Gesellschaft. Grundriss der verstehenden Soziologie. Tübingen: Mohr Verlag. 